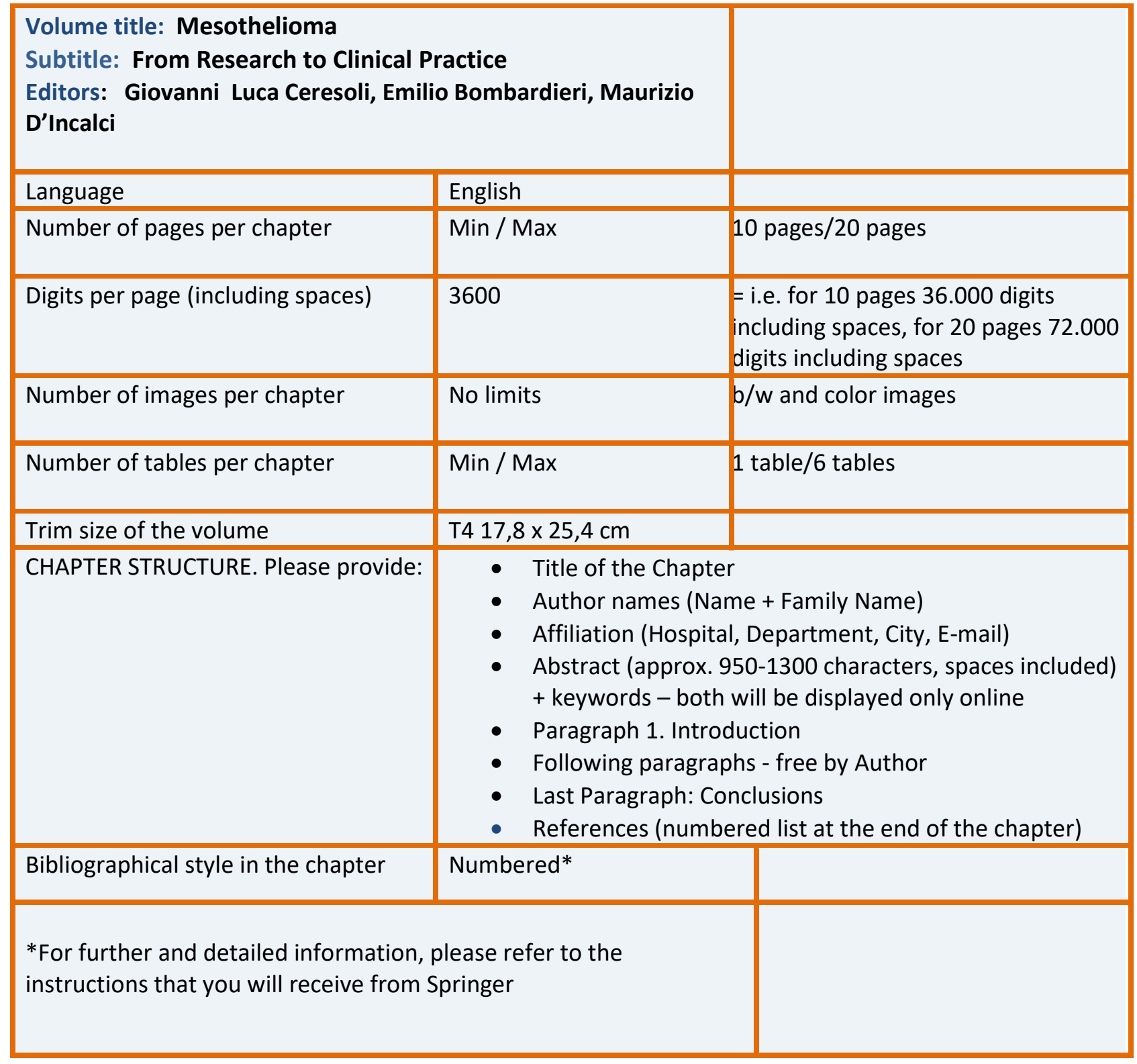

- Title of the Chapter

- Author names (Name + Family Name)

- Affiliation (Hospital, Department, City, E-mail)

- Abstract (approx. 950-1300 characters, spaces included) + keywords - both will be displayed only online

- Paragraph 1. Introduction

- Following paragraphs - free by Author

- Last Paragraph: Conclusions

References (numbered list at the end of the chapter) 
Chapter title:

\section{Measuring malignant pleural mesothelioma}

Authors:

Anna K Nowak ${ }^{1}$ and Samuel G Armato III

Author affiliations:

1. National Centre for Asbestos related Diseases and Medical School, University of Western Australia. 35 Stirling Hwy Nedlands WA 6009. Email: anna.nowak@uwa.edu.au

2. Department of Radiology, The University of Chicago, 5841 South Maryland Ave., MC 2026 Chicago, Illinois, USA 60637. Email: s-armato@uchicago.edu 


\section{Abstract:}

Measurement of malignant pleural mesothelioma poses significant challenges, but tumor measurement is important for clinical trials and patient prognostication. Whilst anatomical T staging of mesothelioma currently incorporates only the extent of tumor invasion into adjacent structures, tumor burden is emerging as potentially important in this context and is the subject of current research. Assessment of the rate of change, or growth, of mesothelioma is not commonly used in clinical research, but this parameter is often informally considered in clinical practice; future research may reveal rate of tumor growth as a potentially informative prognostic indicator. Finally, the most common role for the measurement of mesothelioma is the assessment of treatment response, either in routine clinical practice or in clinical trials. Modified RECIST (mRECIST) for mesothelioma developed a process for measuring mesothelioma that is more appropriate for the unique growth pattern of this tumor, involving measurement of tumor thickness perpendicular to the chest wall or mediastinum. This process has recently been updated as mRECIST 1.1 to incorporate results of new research and to align with RECIST 1.1; mRECIST 1.1 should be used for the next generation of clinical trials in this disease.

\section{Keywords:}

Mesothelioma

RECIST

Tumor measurement

Response criteria

Tumor volume

Prognosis

Staging 


\section{Introduction}

Measurement of the volume or bulk of tumor has three key purposes. Firstly, when tumor bulk is measured at one timepoint, such as at the time of presentation, it may provide prognostic information. Secondly, when tumor bulk is measured longitudinally, the rate of change may also refine prognostication. Thirdly, tumor bulk measured longitudinally in the context of treatment provides a measure of treatment efficacy, and indirectly, additional prognostic information incorporating information from response or progression on treatment. Hence, measurement of disease underpins important activities which inform clinical trials and epidemiological research - robust staging and measurement of response in groups of patients and determine individual patient care (assessing prognosis and evaluating treatment effectiveness). This chapter will cover the history and current status of measurement of mesothelioma for these purposes and will highlight the most important challenges and areas for further research.

Measuring malignant pleural mesothelioma has posed many difficulties for radiologists, researchers and clinicians. The goal of most tumor measurement, as stated above, is to elicit a metric that can represent a patient's tumor bulk. Whilst most non-mesothelioma tumors grow in a somewhat spherical morphology from an initial nidus of tumor (Figure 1), malignant pleural mesothelioma grows as a rind around the interior of the chest wall and the exterior of the lung, forming a circumferential plaque that is rarely uniform and may involve adjacent structures (Figure 2). Although the volume of a spherical lesion can be approximately quantified through simple mathematics, clearly this is a more difficult proposition for pleural mesothelioma. Furthermore, there are a number of potential confounders when attempting to measure pleural mesothelioma. For example, it may be difficult to distinguish atelectasis, pleural scarring, pleural plaques, or effusion from tumor rind, particularly when pleural effusion is loculated or dense and may appear very similar in density to pleural tumor. These challenges caused by the growth pattern of mesothelioma and the similar density of adjacent structures will impact any form of tumor measurement in this disease.

\section{Measuring tumor for cancer staging}

Cancer staging aims to stratify patient survival by using anatomical tumor characteristics to describe the bulk of the disease and the involvement of organs or other structures. By definition, stage is determined from imaging performed at or around the time of diagnosis, prior to treatment initiation. The relationship among overall cancer burden, metastatic potential, and patient survival is well established in many cancers. Most cancer staging and prognostic systems include some estimate or surrogate of tumor burden within the T stage, often as categories based on 
unidimensional tumor diameter [1]. In many cancers, particularly those that are readily surgically resectable, cancer stage determines subsequent treatment. Cancer stage may also be an important inclusion or stratification factor in clinical trials, and it allows for comparisons among datasets such as treatment or population registries. The AJCC/UICC cancer staging manuals, which undergo revisions every five to seven years, form an internationally consistent platform for staging, with T, N and M descriptors defined for each cancer and each stage. Clinical T categorization, or cT stage, incorporates imaging and physical examination performed prior to the start of any treatment. Historically, mesothelioma T staging has not included any surrogate of tumor volume but has instead detailed the anatomical structures infiltrated by disease, including the chest wall, lung parenchyma, mediastinum and other adjacent structures [2-4]. Certainly, potential surgical management of mesothelioma may be more appropriately determined by anatomical extent of invasion rather than tumor size, but there may be a role for tumor measurements in mesothelioma staging, particularly to aid prognostication where surgical intervention is not proposed (Figure 3a and 3b).

The relationship between tumor burden and survival in mesothelioma has been known for two decades, with a 1997 paper describing the prognostic value of tumor volume in patients undergoing surgical resection. Tumors were measured using three-dimensional reconstructions of chest CT scans, with a measured tumor volume $<100 \mathrm{cc}$ predicting better survival [5]. These findings have been reproduced in a number of studies of CT imaging-derived volume, most commonly in the context of extrapleural pneumonectomy $[6,7]$. Semi-automated quantification of tumor volume using FDG-PET scanning also provides prognostic information, although only when histological subtype is excluded from the model. In patients with epithelioid mesothelioma, total glycolytic volume (TGV), a composite of standardized uptake value intensity and tumor volume, was prognostic on both univariate and multivariate analysis [8]. These findings have been confirmed using a similar methodology that incorporated volumetric aspects with intensity of FDG uptake [9]. Hence, there is substantial evidence for the importance of tumor volume as a prognostic factor in the context of surgery, chemotherapy, and supportive care.

In an effort to provide a stronger evidence basis for the staging of mesothelioma, the International Association for the Study of Lung Cancer (IASLC) developed a prospective staging database that collected, in addition to the usual T staging descriptors of anatomical invasion, three unidimensional measurements of pleural tumor thickness. These measurements were prospectively collected for the purpose of this database from 472 of the total 3519 patients. Unidimensional measurements of tumor thickness were acquired to provide a semi-quantitative surrogate of tumor 
bulk. An important strength of this approach was its simplicity as a technique that could be readily applied in any setting, including when the software, expertise or time for CT-based volumetry was not available. Measurements of pleural tumor were obtained perpendicular to the chest wall or mediastinum at the point of maximum tumor thickness, with one measurement acquired each in the upper, middle and lower third of the thorax (Figure 4a and 4b)[10]. Individual pleural tumor thickness measurements ranged from 0 to $153 \mathrm{~mm}$, and the median thickness increased from the upper to the middle zone and from the middle to the lower zone. These pleural thickness measurements correlated with $\mathrm{T}$ stage categories--as the mean sum of the pleural thickness increased, so did the T stage. These exploratory data were statistically examined in three ways: using the maximum of the three thickness measurements, summing the three measurements, and ranking the measurement sum by quartile to identify prognostic cutpoints. Survival decreased as quartile of summed pleural thickness measurement increased, with the median survival of the lowest quartile $(<16 \mathrm{~mm})$ being 23.4 months, and the median survival of the highest quartile $(>50 \mathrm{~mm})$ being 13.2 months $(p=0.005)$. Data-driven cutpoints of summed measurement below $13 \mathrm{~mm}, 13-60 \mathrm{~mm}$, and above $60 \mathrm{~mm}$ sum also stratified for survival $(p=0.0001)$, and increasing thickness category was associated with increased clinical T category, nodal stage, and overall stage. Even a single data-driven cutpoint for maximal tumor thickness had prognostic significance, with patients in whom no measured tumor thickness exceeded $5.1 \mathrm{~mm}$ having a median survival of 24.2 months, and those in whom any pleural thickness exceeded $5.1 \mathrm{~mm}$ having a median survival of 17.7 months $(p=0.014)$. Moreover, the coarse categorization of tumor morphology into 'minimal', 'nodular', and 'rindlike' yielded a significant difference in survival between those categorized as having minimal disease and those considered to have nodular or rindlike tumor (Figure 5) (18.2 vs. 14.5 months, respectively).

Although these IASLC mesothelioma staging project data provide initial evidence to support the importance of tumor bulk in mesothelioma staging, there is currently insufficient evidence to support a move towards measurement-based staging, and T staging for now remains based on anatomical invasion[10]. Nevertheless, the next iteration of the IASLC mesothelioma prospective staging database will again include unidimensional measurements as a surrogate for tumor bulk and will incorporate a pilot study on volumetric measurements. These data are expected to be collected over the next three years.

Further support for the relationship among unidimensional measurement of thickness, tumor volume, and survival was provided in a recent publication that examined these questions in patients undergoing radiotherapy on the SMART 
protocol prior to definitive surgery for mesothelioma [11]. The investigators obtained three structured measurements from each of the mediastinal, chest wall, and diaphragmatic tumor, yielding a total of nine unidimensional tumor measurements. Tumor volume was estimated from the gross tumor volume derived from a radiation boost volume calculation. The total of these nine measurements correlated significantly $(p<0.0001)$ with tumor volume. The thickness of diaphragmatic tumor was most strongly associated with time to recurrence $(p<0.0001)$ and survival $(p=0.001)$, whilst the association with both was weaker for mediastinal tumor thickness and absent when only chest wall thickness was considered.

Hence, evidence clearly points to the prognostic value of tumor volume, or surrogates of tumor volume such as unidimensional measurements, in mesothelioma. It remains unclear as to whether tumor volume or other measurements will be more strongly prognostic than the current T stage and whether these measurements should be incorporated into T staging in addition to, or instead of, descriptors of anatomical invasion. For routine clinical practice, however, the simplicity of unidimensional measurements is clearly attractive.

\section{Measuring tumor to assess response to treatment}

Tumor response assessment underpins a number of key outcome measures in clinical trials. Not only is objective radiological response a surrogate outcome measure for the biological activity of treatments, but progression-free survival, time to progression, and duration of response are time-to-event measures that also require a robust and validated method of defining response and progression. The first widely used tumor response criteria were the WHO response criteria, which were very poorly suited to the unique growth pattern of pleural mesothelioma. The WHO response criteria were most suited to measuring lesions with well-defined bi-dimensional axes, with each lesion measurement comprising the product of the longest diameter of the lesion and its longest perpendicular diameter[12]. Lesion measurements then were summed to produce a total baseline tumor measurement, and these measurements were repeated at each imaging timepoint. A partial response was defined as a $50 \%$ decrease in the sum of these measurements with respect to baseline. Although unidimensional measurements were allowed, a partial response also required a $50 \%$ decrease in any unidimensional measurement, equating mathematically to a $75 \%$ decrease in the sum of the products of perpendicular diameters. Hence, this measurement system, if used for unidimensional measurements in mesothelioma, required a correspondingly greater reduction in tumor to be considered a 'partial response' and may have contributed to the historical lack of measured chemotherapy efficacy in this disease. 
In 2000, the RECIST 1.0 criteria for assessment of tumor response were developed, an important modification of which was the use of unidimensional tumor measurements only [13]. Unidimensional measurements have been theorized to be more closely related to cell kill by chemotherapy than the bidimensional product, at least with respect to spherical tumor masses [14]. There is also agreement between the sum of the product of diameters and the sum of unidimensional measurements for spherical tumors [14], although others identified discordance between response categories allocated using WHO vs. RECIST 1.0 criteria [15]. Nevertheless, it is important to remember that the unidimensional measurements of RECIST 1.0 evolved from the measurement of essentially spherical lesions and assumed relatively symmetrical changes in all tumor diameters.

RECIST 1.0 is limited in its application to mesothelioma. The unit of measurement is the 'lesion', and given that mesothelioma often comprises one contiguous tumor mass, there is no guidance as to which part of this mass would be considered a 'lesion' and whether the mass could be measured in more than one area. Furthermore, the requirement to record 'all' other lesions or sites of disease as non-target lesions imposes a difficult documentation burden on those measuring mesothelioma. RECIST 1.0 requires the measurement of each lesion's 'longest diameter', which is clearly problematic in mesothelioma as the growth of mesothelioma usually follows the curvature of the chest wall, with no clear endpoint to the longest diameter (Figure 6a). Furthermore, when mesothelioma responds to therapy, that response usually demonstrates as a reduction in tumor thickness, rarely as a reduction in the extent of tumor along the chest wall or mediastinum (Figure 6b). The 'longest diameter' could also be applied to measurements of structures of fixed length, for example, tumor infiltrating the pulmonary fissures or tumor between two fixed structures such as the carina and thoracic vertebrae; however, clearly this presentation would again be inappropriate for assessment by longest diameter, as any reduction in tumor burden would reduce the thickness of pulmonary fissure involvement, for example, rather than the longest diameter of the tumor.

Following the publication and adoption of RECIST 1.0, shortcomings of these criteria as applied to mesothelioma were rapidly highlighted in the literature [16-18]. However, prior to the development of RECIST 1.0, an adaptation of response criteria that utilized unidimensional measurements of the tumor rind thickness had been described and used in two phase II clinical trials $[19,20]$. A similar set of criteria also was adopted for the pivotal randomised phase III clinical trial of cisplatin and pemetrexed, which first demonstrated benefits of chemotherapy in advanced mesothelioma and 
promoted the utility of incorporating unidimensional measurements of tumor rind thickness as a surrogate for overall survival benefit [21]. Widespread acceptance of the results of this important clinical trial laid the foundation for acceptance of modified RECIST for mesothelioma, published in 2004 [22].

\section{RECIST modified for mesothelioma}

Modified RECIST for mesothelioma (mRECIST) was published as a research paper and subsequently became the de-facto standard methodology for response assessment in mesothelioma, a fact often overlooked when noting gaps in the measurement and response assessment approaches outlined in this paper [22]. In the context of specific guidance on implementation of tumor measurement protocols, mRECIST did not include, or did not specify in detail, some key points. Essentially, mRECIST for mesothelioma did not propose a new set of response criteria and was implicitly intended to align with RECIST 1.0 when considering issues such as minimum measurable disease, categories of response, and handling of non-pleural lesions; however, it described a set of guidelines around how to obtain tumor measurements in this disease. Most notably, mRECIST proposed two measurements perpendicular to the chest wall or mediastinum at each of three levels (CT sections) to capture tumor thickness in the affected hemithorax (Figure 7a-c). The sum of these measurements then became the unidimensional pleural measurement for any given CT scan, with the sum of the unidimensional measurements of any additional non-pleural lesions being treated as per RECIST 1.0 and added to the pleural measurement. Criteria for response (a reduction of at least $30 \%$ in the summed tumor thickness measurements) and progression (a summed measurement increase of at least $20 \%$ ) were unchanged by mRECIST. Despite some ambiguities, mRECIST has generally been applied as intended and has now been widely used in mesothelioma clinical trials for over a decade [23-29]. Although the extent of reduction in unidimensional measurements that should be considered 'partial response' in mesothelioma has been debated, the original mRECIST paper did demonstrate improved patients outcomes with a $30 \%$ reduction in unidimensional measurements. More recently, Labby et al. used tumor thickness as a continuous variable in an independent cohort and validated that change in mRECIST-acquired tumor thickness measurements was independently associated with patient prognosis [30].

\section{Updating RECIST modified for mesothelioma}

In 2009, RECIST was updated to version 1.1, with a suite of publications detailing not only the updated guidelines but also reporting in detail the research supporting the proposed changes [31-38]. Notable changes included reducing the number of lesions to be measured per organ, providing specific guidelines for the measurement of pathological lymph 
nodes, changes to requirements for confirmation of response, and incorporation of a minimum increase in tumor measurement when determining response in order to mitigate the risk of overcalling progression based on small changes in tumor measurement. These changes had not yet been applied to mesothelioma, as mRECIST remained the standard response guidelines in this disease. Some elements of RECIST 1.1 were intuitively appropriate to incorporate in the mesothelioma setting; for example, measurement of pathologically involved lymph nodes had excellent validity for use in mesothelioma. The requirements for confirmation of response only when response was a primary endpoint were also relevant; however, to consider a reduction in the number of lesions measured from an involved hemithorax from six (per mRECIST) to two (per RECIST 1.1) would substantially reduce the representativeness of any measured area of tumor.

To clarify ambiguities in mRECIST and incorporate important and relevant updates from RECIST 1.1, modified RECIST 1.1 for mesothelioma (mRECIST 1.1) was developed [39]. This paper provides more detailed guidance on the application of measurements in mesothelioma, adds updated recommendations in the context of RECIST 1.1, and incorporates intervening research, as well as clarifications from a decade of experience. Aspects that required specific clarification included definition of minimal measureable disease and measurable lesions, more guidance around location of measurements and descriptions of non-measurable pleural disease, and incorporation of specific considerations around pathological lymph nodes, non-pleural disease, bilateral pleural disease, and progressive disease.

RECIST 1.0 and 1.1 conceptualize the 'lesion' as the unit to be measured, but this concept is difficult to apply to mesothelioma. Whilst many other tumors are discrete foci that can be measured in a longest dimension, the growth pattern of mesothelioma as a circumferential sheet or rind around the lung means that a patient's tumor could actually comprise one continuous tumor mass, potentially a single 'lesion', extending through multiple levels of the hemithorax and across multiple CT sections. However, to consider that the complexities of tumor growth or response in mesothelioma could be captured by one measurement from this single lesion is overly simplistic. Modified RECIST 1.1 hence formalizes the concept of a 'measurement site' on the pleura, allowing the observer to select a number of appropriate measurement sites irrespective of the number of separate physical lesions. The observer first selects CT sections with the greatest pleural thickness and identifies sites on these sections that are most suitable to reproducible longitudinal measurements. When measuring tumor thickness, the observer must select a point on the outer margin of the tumor to initiate the measurement and then measure in a direction that best captures the thickness of the tumor 
at the site, extending the measurement to the inner margin of the tumor [40]. Ideally, these measurements would be made using a computer interface and will be, as per mRECIST, perpendicular to a tangent to the curve of the pleura on the chest wall or mediastinum. Even when the same point on the outer tumor margin is selected, different observers may construct measurement lines either in different directions or with differing interpretations of the inner margin of the tumor, thus leading to interobserver variability $[40,41]$. Hence, in order to reduce variability, mRECIST 1.1 recommends that once a baseline measurement has been obtained at a specific measurement site, all subsequent measurements at that site should be oriented in the same direction. Furthermore, the same image display parameters should be used at each timepoint, which may provide more consistency in selecting the inner margin of the tumor. mRECIST 1.1 also suggests that the same observer acquire measurements across timepoints. To maximize temporal consistency, even when the same observer is obtaining measurements sequentially, it is highly recommended that observers capture annotated images for use as a visual reference when future measurements are acquired.

The original mRECIST did not explicitly state the minimum tumor thickness measurement but was intended to accord with the RECIST 1.0 definition of $10 \mathrm{~mm}$ as the minimum measurable disease thickness. The concept underpinning this minimum lesion size recommendation was that the minimum size for a measurable lesion should be twice the CT scan section thickness, which was $5 \mathrm{~mm}$ at the time RECIST was published; despite section thicknesses of 1 to $3 \mathrm{~mm}$ now standard on contemporary CT scanners, this $10-\mathrm{mm}$ recommendation was not updated by RECIST 1.1. CT Scanner resolution combined with the typical presentation of mesothelioma tumor as a sheet that extends across multiple contiguous axial CT sections seemed to suggest that this size recommendation could be reduced; however, as the size of an object to be measured decreases, the variability of measurement increases, thus increasing the chance of incorrect classification of response [42]. As an intervening study in mesothelioma measurement demonstrated that observer variability was acceptable down to tumor thickness measurements of 5 or $7.5 \mathrm{~mm}$ [43], mRECIST 1.1 now proposes a decrease in the requirement for minimally measurable tumor thickness from 10 to $7 \mathrm{~mm}$. This change has the potential to increase the proportion of patients with earlier disease who may become eligible for clinical trials based on measurable tumor and will redress, in part, the disadvantage that patients with mesothelioma face through the 10-mmthickness requirement, which equates to a very substantial tumor burden in this disease due to its unique morphology [44]. This change may also increase the number of sites available for measurement in clinical trial participants, potentially decreasing measurement variability through incorporation of more discrete measurement sites. 
mRECIST 1.1 also clarifies the number of sites that should be measured for assessment of response. Whilst RECIST 1.0 proposed measurement of up to 10 lesions in total with up to 5 in any one organ, RECIST 1.1 reduced the number of lesions to be measured to two per organ. mRECIST for mesothelioma originally specified the selection of six measurement sites but was ambiguous as to whether all six sites were required or whether the six sites represented a maximum number. mRECIST 1.1 for mesothelioma now specifies that up to six pleural measurement sites may be selected and that the measurement at each site must meet the criterion for minimally measurable disease. Each $\mathrm{CT}$ section for measurement is ideally selected on the basis of measurement reproducibility, with anatomic landmarks being readily identifiable for matching axial measurement levels in scans at subsequent timepoints. Nevertheless, mRECIST 1.1 also recognizes that the presence of measurable tumor is of primary importance. Because sites superior to the level of the left atrium are less vulnerable to the impact of inspiratory effort, and those below the level of the aortic arch reduce the impact of volume averaging and pleural curvature, these considerations should be incorporated into selection of measurement sites.

mRECIST 1.1 specifically addresses circumstances such as measurement of bilateral disease, non-pleural lesions, and measurement of nodal disease. Bilateral disease should be measured as if the pleura is a single organ, with a maximum of six pleural measurement sites distributed across both pleurae. Similarly, non-pleural lesions are handled as per RECIST 1.1; however, the up-to-six pleural measurement sites will be counted as the contribution from one organ (i.e., nominally as two measurements) towards the sum of measurements specified by RECIST 1.1. Furthermore, non-pleural lesions can be considered 'measurable disease', even if no measurable pleural disease is apparent. Nodal disease should be measured unidimensionally as per RECIST 1.1, with any nodes identified as target lesions having a short axis of $\geq 15$ $\mathrm{mm}$ and with the nodal short-axis measurement added to the overall measurement of tumor burden. mRECIST 1.1 acknowledges that some nodal sites, for example internal mammary nodes and intercostal nodes, are unlikely to be seen at all unless pathological; however, at the moment there is insufficient data to make any recommendations that differ from RECIST 1.1.

Because mesothelioma is often circumferential, there may be many areas of tumor that cannot be incorporated into specific measurement sites or may not meet criteria for measurable disease. The morphology of mesothelioma does not allow for these areas to be individually noted, hence mRECIST 1.1 allows for other foci of disease to be described as a whole with descriptive terms such as 'extensive pleural thickening', 'extensive pleural nodularity', or 'circumferential 
pleural thickening'. There is no expectation that numerous individual pleural lesions be specifically identified as nontarget lesions.

Under mRECIST 1.1, tumor response criteria for partial and complete response and stable disease mirror those of RECIST 1.1, as does a requirement for partial or complete response to be confirmed by a follow-up scan at least 4 weeks later; however, whilst progressive disease still requires an increase in the summed measurement of at least $20 \%$ over the nadir measurement, an absolute increase of the summed measurement of at least $5 \mathrm{~mm}$ over the nadir summed measurement is also required, consistent with RECIST 1.1. Assessment of 'unequivocal' new lesions also requires careful review of adjacent CT sections to ensure that the "new" lesion has not been displaced, for example, from an adjacent section with change in inspiratory effort or thoracic contraction. Regarding a measurement site that demonstrates reduced thickness, RECIST 1.1 specifies a default value of $5 \mathrm{~mm}$ is to be assigned if an actual measurement cannot be acquired; however, given that the sheet-like structure of mesothelioma has one margin that generally abuts normal structures and that the partial volume effect in the axial dimension does not need to be considered, mRECIST 1.1 recommends a default value of $2 \mathrm{~mm}$ if tumor is present at a measurement site but is too thin to accurately measure.

Because mesothelioma is non-spherical, the RECIST response classification criteria may not reflect the same changes in volume for the unidimensional changes that categorise response. This notion has been demonstrated on geometric modelling [44] and also using patient imaging data. In fact, the response criteria that were most highly correlated with survival were a reduction of $64 \%$ in unidimensional measurement for partial response and an increase of $50 \%$ for progressive disease [30]. Nevertheless, until alternative criteria are fully validated in a prospective clinical trial, no changes have been recommended for mRECIST 1.1.

\section{Measurement of mesothelioma in immunotherapy clinical trials}

The advent of immunotherapy has required some reframing of response criteria developed to assess response to chemotherapy. As with other cancers, mesothelioma is the subject of numerous immunotherapy clinical trials, with the key challenge in response assessment being immune-related pseudoprogression. Pseudoprogression is believed to develop when the immunological response to tumor leads to an influx of immune cells, which may result in an apparent increase in the bulk of the tumor as seen on imaging. This process has been considered in a number of modifications to the RECIST criteria, most recently with a consensus-based modification of RECIST 1.1 for immune-based therapeutics published by the RECIST working group[45]. These iRECIST guidelines, which are based on RECIST 1.1 measurements, 
allow patients to continue on clinical trials despite development of apparent new lesions or suspected initial progression of baseline target lesions, which initially would be considered 'unconfirmed progressive disesase' (iUPD). With continued imaging, iUPD can subsequently become 'confirmed progressive disease' (iCPD). This approach allows for patients to continue treatment when there is suspicion of pseudoprogression. iRECIST did not specify any considerations around pleural mesothelioma; however, mRECIST 1.1 recommends that the general principles of iRECIST be adopted for immunotherapy clinical trials in which pseudoprogression or delayed response may occur. Each clinical trial protocol should include adequate specific guidance on the application of iRECIST, informed by the general principles of mRECIST 1.1.

\section{Incorporating FDG-PET-CT into response assessment for mesothelioma}

${ }^{18} \mathrm{~F}-\mathrm{FDG}-\mathrm{PET} / \mathrm{CT}$ is an important cancer imaging modality that has proven useful in response assessment in other cancers. FDG-PET and FDG-PET-CT have been studied in response assessment in pleural mesothelioma; however, PET-based imaging has not been well validated as a surrogate measure of outcome in large patient cohorts or in a prospective randomised clinical trial [46-49]. There are also important limitations to the measurement of response using FDG-PET, most notably the difficulty in interpreting changes in FDG uptake and SUV in the context of postoperative changes, inflammation or infection, or prior pleurodesis [50-52]. mRECIST 1.1 does not currently recommend incorporating FDGPET-CT into measurement of response, and it is likely that the difficulties in applying FDG-PET criteria to a large subgroup of patients who have had pleural surgery or pleurodesis mean that this will not become a validated standard in the future (Figure 8). Other PET tracers such as FLT-PET have been tested but also suffer from limitations [53].

\section{Using CT volumetry in measurement of pleural mesothelioma}

The potential of volumetry in the assessment of tumor response has also been studied and, if consistent and validated, would render the need for tumor measurement guidelines obsolete. Tumor volume also has potential use in staging. Clearly, the clinical use of change in volume would require a different response and progression metric. Mathematically, the RECIST response criteria, 30\% reduction in tumor diameter for partial response and $20 \%$ increase in tumor diameter for progressive disease, equate to a $66 \%$ reduction in tumor volume and a $73 \%$ increase in tumor volume, respectively, using the spherical model for which these criteria were derived [44]. When these proposed volumetric 'response criteria' were applied to pre- and post-chemotherapy CT scans in one study, there was no significant difference among three readers in derived tumor volume $(p>0.42)$ and a high intraclass correlation coefficient (0.99) for agreement among 
readers in response category [54]. This was not the case for response categories derived from mRECIST measurements, for which there was poor agreement among three readers. Other investigators have found significant variability in tumor volume measurements for mesothelioma [55]. Although there is an acknowledged association between mesothelioma tumor volume and survival [Harvey article and other Ritu article], the use of response categories translated directly from unidimensional measurements has not been validated, nor have any new volumetric response metrics been developed with sufficient clinical validation to be used in clinical trials or clinical decision making[56]. Consistent application of tumor volume measurements would require use of the same image analysis software, patient set-up, and image acquisition parameters; however, it remains difficult to standardize radiologist perception. Even in the simpler context of lung nodule volumetry, these issues have been challenging to standardize [57].

Measurement of tumor volume in mesothelioma is very challenging. The difficulties in distinguishing mesothelioma from adjacent structures, pleural effusion, and atelectasis are not confined to the human eye; computerized systems also struggle to differentiate between tumor and other structures that may mimic the texture and imaging characteristic of tumor[58]. Correlation between physical tumor bulk of post-operative specimens and CT-obtained tumor volumes was also lower than expected [59]. Indeed, an advantage of selecting individual representative sites of tumor for measurement, as per mRECIST, is that ambiguous areas and regions that are difficult to measure with confidence can be avoided. Furthermore, there are no tumor volume measurement software systems that do not require radiologist expertise and input; this need would pose a challenge in clinical trials, in which site clinical investigators commonly acquire measurements. MRI volumetry has also been studied, but MRI is more time consuming that CT and remains a more scarce resource in most settings; there has been limited research in this space[60].

It is worth noting that whilst the accuracy of tumor volume measurements as a representation of tumor bulk and change in tumor bulk may, in the future, be superior to unidimensional measurements, the goal of tumor measurement for response is not necessarily accuracy per se, but rather the use of a reproducible, simple, and adequate surrogate of patient outcomes. Taking these considerations into account, mRECIST 1.1 does not recommend the use of tumor volume for the current response criteria and proposes that mesothelioma tumor volumetry remains a research tool at the moment.

\section{Conclusion}


In conclusion, measurement of pleural tumor in malignant mesothelioma is a key part of clinical management and clinical trial interpretation. Clinicians, radiologists, and investigators, however, are challenged by the unique rind-like growth pattern of this disease, which leads to difficulty in applying staging and response criteria that perform well in tumors of a more spherical morphology. Nevertheless, more appropriate measurement guidelines for response have been developed for this disease and are in widespread use in clinical trials. These guidelines recently have been comprehensively updated to harmonize with RECIST 1.1 and to clarify aspects that were ambiguous in the original mRECIST publication. The use of tumor measurement in staging is under investigation, and although preliminary data support the prognostic potential of tumor measurements, they have not yet been formally incorporated into staging in this disease. CT scans remain the mainstay of imaging for tumor measurement in mesothelioma. Techniques such as CT volumetrics are promising research tools but have not yet been incorporated into routine clinical or clinical trial use since optimal, reproducible platforms and techniques remain unclear. MRI and FDG-PET scans have specific limitations in this context and are not routinely used in the measurement of tumor for response assessment. mRECIST 1.1 now should be considered the standard criteria for the assessment of tumor response to treatment in pleural mesothelioma. 


\section{References}

1. Ball, D., et al., Effect of tumor size on prognosis in patients treated with radical radiotherapy or chemoradiotherapy for non-small cell lung cancer. An analysis of the staging project database of the International Association for the Study of Lung Cancer. J Thorac Oncol, 2013. 8(3): p. 315-21.

2. Cancer, A.J.C.o., Cancer Staging Handbook. 7th edition ed, ed. S.B. Edge and D.R. Byrd. 2010, New York: Springer.

3. Rusch, V.W., A proposed new international TNM staging system for malignant pleural mesothelioma from the International Mesothelioma Interest Group. Lung Cancer, 1996. 14(1): p. 112.

4. Rusch, V.W. and D. Giroux, Do we need a revised staging system for malignant pleural mesothelioma? Analysis of the IASLC database. Ann Cardiothorac Surg, 2012. 1(4): p. 438-48.

5. Pass, H.I., et al., Preoperative tumor volume is associated with outcome in malignant pleural mesothelioma. J Thorac Cardiovasc Surg, 1998. 115(2): p. 310-7; discussion 317-8.

6. Olt, G., A. Berchuck, and R.C. Bast, Jr., The role of tumor markers in gynecologic oncology. Obstetrical \& Gynecological Survey, 1990. 45(9): p. 570-7.

7. Gill, R.R., et al., Epithelial malignant pleural mesothelioma after extrapleural pneumonectomy: stratification of survival with CT-derived tumor volume. AJR Am J Roentgenol, 2012. 198(2): p. 35963.

8. Nowak, A.K., et al., A novel prognostic model for malignant mesothelioma incorporating quantitative FDG-PET imaging with clinical parameters. Clinical Cancer Research, 2010. 16(8): p. 2409-17.

9. Klabatsa, A., et al., The association of 18F-FDG PET/CT parameters with survival in malignant pleural mesothelioma. Eur J Nucl Med Mol Imaging, 2014. 41(2): p. 276-82.

10. Nowak, A.K., et al., The IASLC Mesothelioma Staging Project: Proposals for Revisions of the T Descriptors in the Forthcoming Eighth Edition of the TNM Classification for Pleural Mesothelioma. J Thorac Oncol, 2016. 11(12): p. 2089-2099.

11. de Perrot, M., et al., Impact of tumour thickness on survival after radical radiation and surgery in malignant pleural mesothelioma. Eur Respir J, 2017. 49(3).

12. Miller, A., et al., Reporting results of cancer treatment. . Cancer, 1981. 47: p. 207-214.

13. Therasse, P., et al., New guidelines to evaluate the response to treatment in solid tumors. Journal of the National Cancer Institute, 2000. 92(3): p. 205-16.

14. James, K., et al., Measuring response in solid tumors: unidimensional versus bidimensional measurement. J Natl Cancer Inst, 1999. 91(6): p. 523-8.

15. Mazumdar, M., A. Smith, and L.H. Schwartz, A statistical simulation study finds discordance between WHO criteria and RECIST guideline. J Clin Epidemiol, 2004. 57(4): p. 358-65.

16. van Klaveren, R.J., et al., Inadequacy of the RECIST criteria for response evaluation in patients with malignant pleural mesothelioma. Lung Cancer, 2004. 43(1): p. 63-9.

17. Hillerdal, G., Staging and evaluating responses in malignant pleural mesothelioma. Lung Cancer, 2004. 43(1): p. 75-6.

18. Monetti, F., et al., Inadequacy of the new Response Evaluation Criteria in Solid Tumors (RECIST) in patients with malignant pleural mesothelioma: report of four cases. Lung Cancer, 2004. 43(1): p. 714.

19. Nowak, A.K., et al., A multicentre phase II study of cisplatin and gemcitabine for malignant mesothelioma. British Journal of Cancer, 2002. 87(5): p. 491-6.

20. Byrne, M.J., et al., Cisplatin and gemcitabine treatment for malignant mesothelioma: a phase II study. Journal of Clinical Oncology, 1999. 17(1): p. 25-30.

21. Vogelzang, N.J., et al., Phase III study of pemetrexed in combination with cisplatin versus cisplatin alone in patients with malignant pleural mesothelioma. J Clin Oncol, 2003. 21(14): p. 2636-44.

22. Byrne, M.J. and A.K. Nowak, Modified RECIST criteria for assessment of response in malignant pleural mesothelioma. Annals of Oncology, 2004. 15(2): p. 257-60. 
23. Maio, M., et al., Tremelimumab as second-line or third-line treatment in relapsed malignant mesothelioma (DETERMINE): a multicentre, international, randomised, double-blind, placebocontrolled phase $2 b$ trial. Lancet Oncol, 2017. 18(9): p. 1261-1273.

24. Zalcman, G., et al., Bevacizumab for newly diagnosed pleural mesothelioma in the Mesothelioma Avastin Cisplatin Pemetrexed Study (MAPS): a randomised, controlled, open-label, phase 3 trial. Lancet, 2016(387): p. 1405-14.

25. Kindler, H.L., et al., Multicenter, double-blind, placebo-controlled, randomized phase II trial of gemcitabine/cisplatin plus bevacizumab or placebo in patients with malignant mesothelioma. Journal of Clinical Oncology, 2012. 30(20): p. 2509-15.

26. Krug, L.M., et al., VANTAGE 014: Vorinostat (V) in patients with advanced malignant pleural mesothelioma (MPM) who have failed prior pemetrexed and either cisplatin or carboplatin therapy: a phase III, randomized, doubleblind, placebo-controlled trial. Eur J Cancer, 2011. 47: p. 2-3.

27. Nowak, A.K., et al., A phase $1 \mathrm{~b}$ clinical trial of the CD40-activating antibody CP-870,893 in combination with cisplatin and pemetrexed in malignant pleural mesothelioma. Ann Oncol, 2015. 26(12): p. 2483-90.

28. Nowak, A.K., et al., A phase Il clinical trial of the vascular disrupting agent BNC105P as second line chemotherapy for advanced Malignant Pleural Mesothelioma. Lung Cancer, 2013. 81(3): p. $422-7$.

29. Nowak, A.K., et al., A Phase II Study of Intermittent Sunitinib Malate as Second-Line Therapy in Progressive Malignant Pleural Mesothelioma. Journal Of Thoracic Oncology, 2012. 7(9): p. 1449-56.

30. Labby, Z.E., et al., Optimization of Response Classification Criteria for Patients with Malignant Pleural Mesothelioma. Journal Of Thoracic Oncology, 2012. 7(11): p. 1728-1734.

31. Eisenhauer, E.A., et al., New response evaluation criteria in solid tumours: revised RECIST guideline (version 1.1). European Journal of Cancer, 2009. 45(2): p. 228-47.

32. Bogaerts, J., et al., Individual patient data analysis to assess modifications to the RECIST criteria. European Journal of Cancer, 2009. 45(2): p. 248-60.

33. Schwartz, L.H., et al., Evaluation of lymph nodes with RECIST 1.1. European Journal of Cancer, 2009. 45(2): p. 261-7.

34. Ford, R., et al., Lessons learned from independent central review. Eur J Cancer, 2009. 45(2): p. 26874.

35. Dancey, J.E., et al., Recommendations for the assessment of progression in randomised cancer treatment trials. European Journal of Cancer, 2009. 45(2): p. 281-9.

36. Moskowitz, C.S., et al., A simulation study to evaluate the impact of the number of lesions measured on response assessment. European Journal of Cancer, 2009. 45(2): p. 300-10.

37. Verweij, J., et al., Cancer clinical trial outcomes: any progress in tumour-size assessment? European Journal of Cancer, 2009. 45(2): p. 225-7.

38. Sargent, D.J., et al., Validation of novel imaging methodologies for use as cancer clinical trial endpoints. Eur J Cancer, 2009. 45(2): p. 290-9.

39. Armato, S.G., 3rd and A.K. Nowak, Revised Modified Response Evaluation Criteria in Solid Tumors for Assessment of Response in Malignant Pleural Mesothelioma (Version 1.1). J Thorac Oncol, 2018. 13(7): p. 1012-1021.

40. Armato, S.G., 3rd, et al., Measurement of mesothelioma on thoracic CT scans: a comparison of manual and computer-assisted techniques. Med Phys, 2004. 31(5): p. 1105-15.

41. Armato, S.G., 3rd, et al., Evaluation of semiautomated measurements of mesothelioma tumor thickness on CT scans. Acad Radiol, 2005. 12(10): p. 1301-9.

42. Oxnard, G.R., et al., Variability of lung tumor measurements on repeat computed tomography scans taken within 15 minutes. J Clin Oncol, 2011. 29(23): p. 3114-9.

43. Armato, S.G., 3rd, et al., Observer variability in mesothelioma tumor thickness measurements: defining minimally measurable lesions. J Thorac Oncol, 2014. 9(8): p. 1187-94.

44. Oxnard, G.R., S.G. Armato, 3rd, and H.L. Kindler, Modeling of mesothelioma growth demonstrates weaknesses of current response criteria. Lung Cancer, 2006. 52(2): p. 141-8.

45. Seymour, L., et al., iRECIST: guidelines for response criteria for use in trials testing immunotherapeutics. Lancet Oncol, 2017. 18(3): p. e143-e152. 
46. Veit-Haibach, P., et al., Combined FDG-PET/CT in response evaluation of malignant pleural mesothelioma. Lung Cancer, 2009. Epub ahead of print May 302009.

47. Schaefer, N.G., et al., Response evaluation by CT and FDG-PET/CT in malignant pleural mesothelioma. J Clin Oncol 2008. 26(18S): p. Abs 11098.

48. Ceresoli, G.L., et al., Early response evaluation in malignant pleural mesothelioma (MPM) by total glycolytic volume (TGV) analysis of serial FDG-PET scans. 2008.

49. Francis, R.J., et al., Early prediction of response to chemotherapy and survival in malignant pleural mesothelioma using a novel semiautomated 3-dimensional volume-based analysis of serial 18F-FDG PET scans. Journal of Nuclear Medicine, 2007. 48(9): p. 1449-58.

50. Nowak, A.K., et al., A Novel Prognostic Model for Malignant Mesothelioma Incorporating Quantitative FDG-PET Imaging with Clinical Parameters. Clinical Cancer Research, 2010. 16(8): p. 2409-2417.

51. Kwek, B.H., S.L. Aquino, and A.J. Fischman, Fluorodeoxyglucose positron emission tomography and CT after talc pleurodesis. Chest, 2004. 125(6): p. 2356-60.

52. Genestreti, G., et al., FDG PET/CT Response Evaluation in Malignant Pleural Mesothelioma Patients Treated with Talc Pleurodesis and Chemotherapy. J Cancer, 2012. 3: p. 241-5.

53. Segard, T., et al., FLT PET for response assessment in malignant pleural mesothelioma (MPM) using a semi-automated volume-based region growing algorithm. European Journal of Nuclear Medicine and Molecular Imaging, 2012. 39: p. S457-S457.

54. Frauenfelder, T., et al., Volumetry: an alternative to assess therapy response for malignant pleural mesothelioma? Eur Respir J, 2011. 38(1): p. 162-8.

55. Gill, R.R., et al., North American Multicenter Volumetric CT Study for Clinical Staging of Malignant Pleural Mesothelioma: Feasibility and Logistics of Setting Up a Quantitative Imaging Study. J Thorac Oncol, 2016. 11(8): p. 1335-1344.

56. Armato, S.G., 3rd, et al., Imaging in pleural mesothelioma: A review of the 12th International Conference of the International Mesothelioma Interest Group. Lung Cancer, 2015. 90(2): p. 148-54.

57. Sullivan, D.C., et al., Metrology Standards for Quantitative Imaging Biomarkers. Radiology, 2015. 277(3): p. 813-25.

58. Corson, N., et al., Characterization of mesothelioma and tissues present in contrast-enhanced thoracic CT scans. Med Phys, 2011. 38(2): p. 942-7.

59. Armato, S.G., 3rd, et al., Radiologic-pathologic correlation of mesothelioma tumor volume. Lung Cancer, 2015. 87(3): p. 278-82.

60. Plathow, C., et al., Therapy response in malignant pleural mesothelioma-role of MRI using RECIST, modified RECIST and volumetric approaches in comparison with CT. Eur Radiol, 2008. 18(8): p. 163543. 